\title{
Dynamic versus static fission paths with realistic interactions
}

\author{
Samuel A. Giulian* and Luis M. Robledd ${ }^{\dagger}$ \\ Departamento de Física Teórica, Universidad Autónoma de Madrid, E-28049 Madrid, Spain \\ R. Rodríguez-Guzmán团 \\ Department of Physics and Astronomy, Rice University, Houston, Texas 7r005, USA and \\ Department of Chemistry, Rice University, Houston, Texas 77005, USA
}

\begin{abstract}
The properties of dynamic (least action) fission paths are analyzed and compared to the ones of the more traditional static (least energy) paths. Both the BCPM and Gogny D1M energy density functionals are used in the calculation of the HFB constrained configurations providing the potential energy and collective inertias. The action is computed as in the WKB method. A full variational search of the least-action path over the complete variational space of HFB wave functions is cumbersome and probably unnecessary if the relevant degrees of freedom are identified. In this paper, we consider the particle number fluctuation degree of freedom that explores the amount of pairing correlations in the wave function. For a given shape, the minimum action can be up a factor of three smaller than the action computed for the minimum energy state with the same shape. The impact of this reduction on the lifetimes is enormous and dramatically improves the agreement with experimental data in the few examples considered.
\end{abstract}

\section{INTRODUCTION}

The description of fission in the atomic nucleus is the subject of a renewed interest as a consequence of potential applications both at the level of fundamental science and applications [ [- $[$ ] $]$. The mechanism of fission involves a delicate balance between quantum mechanical effects inherent to the quantum many body problem and the properties of the nuclear interaction that are not well understood yet. Deepening our understanding of fission is therefore important to understand both effects in the dynamics of the atomic nucleus. Fission is also relevant to other areas outside traditional nuclear physics like astrophysics where the understanding of the nucleosynthesis of heavy elements in explosive galactic environments through the r-process is of great relevance. A deeper understanding of fission is also of interest in safe energy production with new generation nuclear reactors or in radioactive waste degradation. The theoretical interpretation of fission is based on two properties of the parent nucleus: the evolution of the energy as the system traverses from its ground state to scission and the inertia associated to the collective motion along that path. To improve in the former, properties of the fission energy landscape as the inner and outer barrier heights have been considered as a physical constraint in the fitting protocol of several energy density functionals (EDFs) [710]. On the other hand, an effort to improve the description of the collective inertias is underway [11, 12]. The gross features of fission can be understood rather well from a microscopic perspective using the mean field Hartree-Fock-Bogoliubov (HFB) theory [13] along with

\footnotetext{
* sam.and.giuliani@gmail.com

$\dagger$ luis.robledo@uam.es

‡ raynerrobertorodriguez@gmail.com
}

effective phenomenological interactions of different kinds. Therefore, it is not surprising the large amount of studies devoted to this subject with Skyrme interactions [10, 14[16], Gogny ones [7, 17 26], based on the relativistic mean field [27-29] or other kind of energy density functionals recently proposed [30, 31].

The traditional paradigm in fission is to describe the path to the scission configuration by looking at the minimum energy in multidimentional energy landscapes. Several deformation parameters associated to multipoles of different orders are routinely used in those calculations. Surprisingly the role played by pairing in the dynamical aspects of the theory has attracted little attention and only recently the uncertainties associated to that degree of freedom in the values of fission observables have been explored [25, 26, 31]. The static (minimum energy) description is an approximation to the more quantal approach where the action of the collective degrees of freedom is the quantity driving the dynamics (the dynamical description). Assuming a static description simplifies the computational problem and in addition, it was argued 32] that both approaches gave equivalent results when shape degrees of freedom alone were considered as collective degrees of freedom (see [33] for a recent result). On the other hand, that pairing is a fundamental ingredient for fission dynamics was already pointed out forty years ago by Moretto and Babinet [34] in a seminal paper where the impact of pairing correlations on the action was analyzed in a simple, yet realistic, model. The main conclusion of that paper was that the competition between the collective inertia decreasing as the square of the inverse pairing gap [6, 35] and the energy increasing as the square of the pairing gap lead to a minimum of the action at a pairing gap twice as large as the one of the minimum energy. The importance of pairing vibrations in nuclear dynamics was empashized by Bés et al [36] in the context of the cranking model and by Gozdz et al [37, 38] in the Generator Coordinate Method (GCM) framework. 
The idea of coupling pairing vibrations to nuclear fission dynamics was pursued further by the Lublin school [38] in a series of papers where realistic fission calculations seeking for the minimum of the action were performed -see [39] for a recent overview and additional references. When pairing was included as dynamical variable they observed reductions of a few orders of magnitude (up to 8 in $\mathrm{Cm}$ isotopes) in the spontaneus fission lifetimes $t_{\mathrm{SF}}$ as compared to the minimum energy results. In nuclei with higher and broader barriers (like the ones considered here) the reduction is expected to be larger.

In this paper we study the predictions for spontaneous fission lifetimes when considering the minimum action scheme instead of the minimum energy one. We will consider pairing as a relevant degree of freedom in the parameter space used in the minimum action search. Calculations with effective, yet realistic, interactions including finite range ones (Gogny D1M [40, 41]) and pure energy density functionals like the BCPM functional [30] will be considered. We will restrict ourselves to several isotopes of uranium where experimental data are available. The main reason for this choice is the large discrepancies observed between theory and experiment and due to very high and wide fission barriers.

\section{METHODS}

The path from the ground state to the scission point is assumed to be driven by the least action principle, where the action is computed as

$$
S=2 \int_{a}^{b} d s \sqrt{2 B(s)\left(V(s)-E_{0}\right)} .
$$

The variable $s$ is used to parametrize the fission path, and all the relevant collective degrees of freedom depend upon it. The inertia is given by the general expression

$$
B(s)=\sum_{i j} B_{i j} \frac{d q_{i}}{d s} \frac{d q_{j}}{d s},
$$

where the $q_{i}(s)$ are the values along the fission path of the relevant degrees of freedom like the axial quadrupole $\left(Q_{20}\right)$, octupole $\left(Q_{30}\right)$ and hexadecapole $\left(Q_{40}\right)$ moments and in our case the particle number fluctuation $\Delta N^{2}$. The $B_{i j}$ are the collective inertias for each pair of degrees of freedom and computed using the perturbative cranking approximation [12, 42]. Both the expressions obtained in the Adiabatic Time Dependent HFB (ATDHFB) or the Generator Coordinate Method (GCM) approximations will be used. The potential $V(s)$ is given by the HFB energy with corrections from beyond mean field effects included. The most important is the rotational energy correction $\epsilon_{\mathrm{ROT}}\left(Q_{20}\right)$ which is the correlation energy gained by restoring angular momentum quantum numbers. It is proportional to deformation and can reach several $\mathrm{MeV}$. It therefore has the potential to reduce the fission barrier heights substantially. It has been computed using a well performing approximation to the exact quantity [43]. Less important for fission is the zero point energy correction associated to quantum fluctuations of the collective $Q_{20}$ degree of freedom $\epsilon_{0}\left(Q_{20}\right)$. Details on how these quantities are evaluated are given in 31] They are computed with the constrained Hartree-Fock-Bogoliubov (HFB) wave functions obtained with state-of-the-art semi-phenomenological energy density functionals of the Gogny and BCP type. Axial symmetry is preserved in the calculation but reflection symmetry is allowed to break at any stage of the calculations. Finally, the computer code HFBaxial [44] has been used.

For this exploratory calculation we have made several simplifying assumptions:

1. Separated proton and neutron pairing correlations are not considered. Instead, a single constraint in the total particle number fluctuation is considered. This simplification reduces the computational cost by a factor of a few tens (depending on the number of $\left\langle\Delta N^{2}\right\rangle$ values considered).

2. The fission path is parametrized by the mass quadrupole moment. As customary in fission calculations based in the minimum energy principle the coupling with other degrees of freedom in the evaluation of the inertia Eq. (2) is neglected and only the quadrupole inertia is considered.

3. As a consequence, the minimum action path does not require for its determination of sophisticated linear programming techniques. It reduces to a simple minimization for fixed quadrupole moments of the integrand in the action considered as a function of the other degrees of freedom.

The constrained HFB wave functions $\left|\varphi\left(Q_{20},\left\langle\Delta N^{2}\right\rangle\right)\right\rangle$ are obtained using an approximate second order gradient method [45] with the HFBaxial code [44]. Two EDF will be used in the calculation to make sure that the conclusions are independent of the details of the nuclear interaction. First we use the Gogny [40] force with the D1M [41] parametrization, whose main characteristic is its finite range that allows a consistent treatment of pairing correlations using the same two body force that determines de particle-hole Hartree- Fock potential. Altough D1M was specifically tailored to describe binding energies, a series of papers [25, 26, 46 -49] have shown that D1M provide good results for many low energy nuclear observables. The other EDF used is the recently proposed BCPM EDF [30] with its density dependent pairing and effective mass equal to one. Again BCPM has been fitted to reproduce binding energies but has proved to produce consistent low energy results in a variety of cases [30]. 

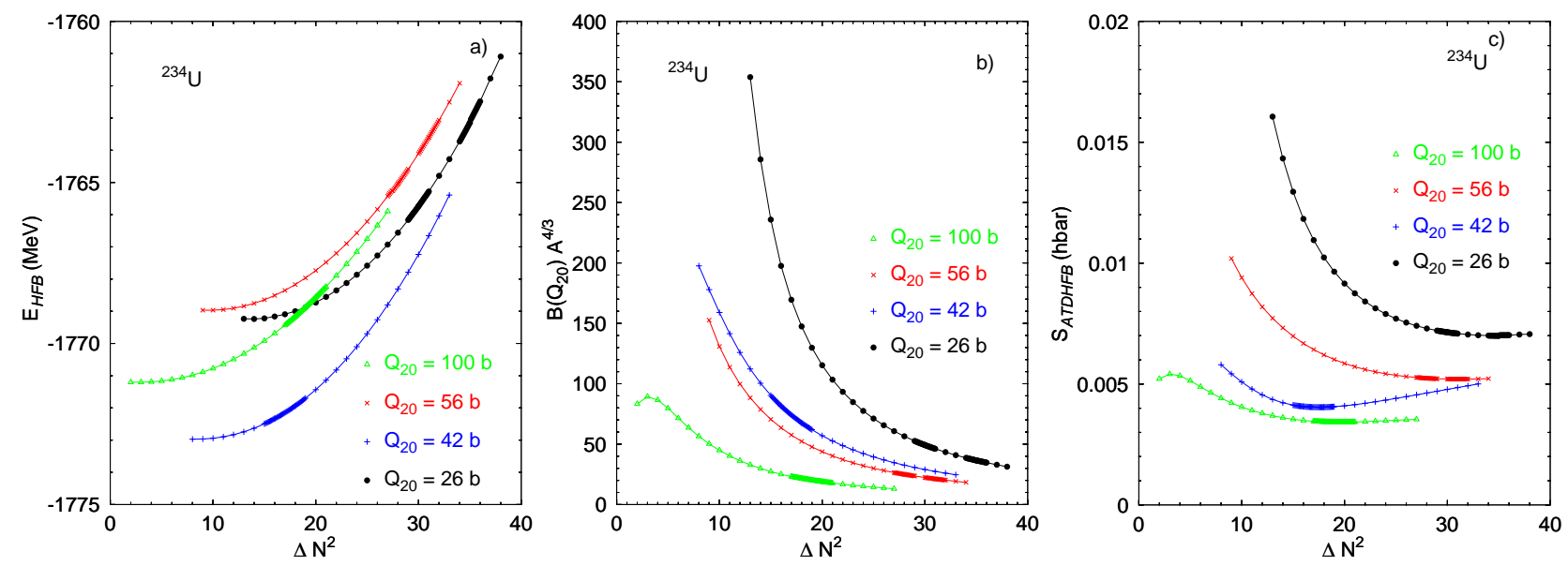

Figure 1. The HFB energy, panel a); the collective inertia, panel b) and the action, panel c) are represented as a function of particle number fluctuation $\Delta N^{2}$ for several relevant $Q_{20}$ values (given in barns).

\section{RESULTS}

To illustrate the procedure we will focus on the nucleus ${ }^{234} \mathrm{U}$ whose fission properties were thoroughly studied in [31] using the BCPM and Gogny D1M functionals. For both functionals, the nucleus presents its ground state minimum at $Q_{20}=12 \mathrm{~b}$. Also for both functionals, the first fission barrier is located at $Q_{20}=26 \mathrm{~b}$, the fission isomer minimum is at $Q_{20}=42 \mathrm{~b}$ and the second fission barrier is at $Q_{20}=62 \mathrm{~b}$. As discussed thoroughly in [31] the main difference between the two functionals is the inertia that is roughly three times larger for BCPM. This is probably a consequence of its larger effective mass and the different pairing interactions used.

To find the $\left\langle\Delta N^{2}\right\rangle$ value minimizing the action we carry out constrained HFB calculations starting at the $\left\langle\Delta N^{2}\right\rangle$ value minimizing the energy for each $Q_{20}$ value of the fission path. In this way we obtain curves for the relevant physical quantities as a function of $\left\langle\Delta N^{2}\right\rangle$ for each value of $Q_{20}$. An example of such curves is shown in Fig. 1 for the $Q_{20}$ values corresponding to the first and second fission barriers, the fission isomer and at $Q_{20}=100 \mathrm{~b}$ as a characteristic value for very large elongations. We observe in panel a) an almost parabolic behavior for the energy as a function of $\left\langle\Delta N^{2}\right\rangle$ with the minimum located at the self-consistent solution. In panel b) the decrease of the inertia with the inverse of $\left\langle\Delta N^{2}\right\rangle^{2}$ (corresponding to the law $\left.B \approx 1 / \Delta^{2}[\underline{6}, 35]\right)$ is noticed. Finally, the integrand in the action $S$ of Eq. (2), computed with the ATDHFB version of the inertia is plotted in panel c). It shows a minimum at rather large $\left\langle\Delta N^{2}\right\rangle$ values as compared with the ones of the minimum energy solution. The minimum value of the action integrand does not coincide with the value obtained (selconsistently) from the minimization of the energy, being the latter one up to a factor three larger than the true minimum. This large quenching reduces considerably the action Eq. (2) that appears in the exponential of the WKB formula. There- fore, the impact on the $t_{\mathrm{SF}}$ values is large. In this case we obtain $0.18 \times 10^{23} \mathrm{~s}$ and $0.21 \times 10^{19} \mathrm{~s}$ depending on the choice of the collective mass, ATDHFB or GCM respectively. Those values have to be compared with the ones obtained minimizing the energy, namely $0.81 \times 10^{43}$ and $0.70 \times 10^{30} \mathrm{~s}$. We observe a reduction of 20 and 11 orders of magnitude that brings the theoretical predictions in much closer agreement with experiment. Another beneficial side effect of the action minimization is that results are much less sensitive to the approach used to compute the inertias. This remarkable reduction was observed in previous calculations [39] by the Lublin group in other heavier nuclei.

The minimum action solutions have $\left\langle\Delta N^{2}\right\rangle$ values much larger than the minimum energy configurations and therefore have stronger pairing correlations. In [25, 26, 31] we analyzed the impact on $t_{\mathrm{SF}}$ of increasing the pairing strength. We observed a strong dependence of the results for $t_{\mathrm{SF}}$ with small changes on the pairing strengths. We have repeated those calculations but now computing the lifetimes using the minimum action principle. The results obtained with BCPM [31] (the ones for Gogny D1M are similar [25]) are show in Fig. 22 as a function of the factor $\eta$ used to modify the pairing strength.

We observe that the strong dependence of $t_{\mathrm{SF}}$ with $\eta$ in the minimum energy calculation gets severely reduced in the minimum action case. This is not so surprising as in the minimum action case we are already exploring the amount of pairing correlations in the search for the minimum action configuration. This search can compensate somehow the changes induced by the varying interaction strength. It is also noteworthy to mention that now the ATDHFB and GCM results are much closer than in the minimum energy case.

Recently, fission calculations based on the minimum action principle and using one of the Skyrme variants have been presented. The variables considered are the axial $Q_{20}$ and triaxial $Q_{22}$ multipole moments. The main 


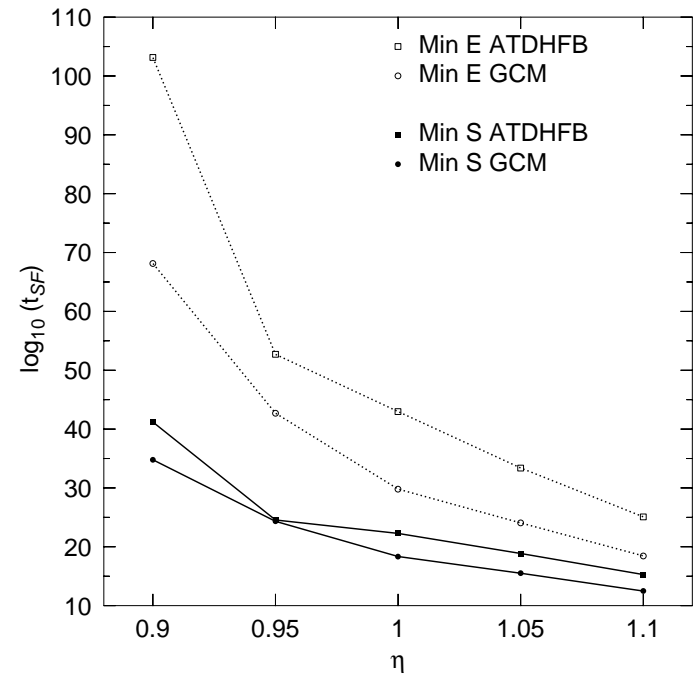

Figure 2. Spontaneous fission lifetimes of ${ }^{234} \mathrm{U}$ obtained with different approaches as a function of the $\eta$ factor multiplying the pairing interaction.

\begin{tabular}{lcc}
\hline \hline Method & $t_{\mathrm{SF}}($ ATDHFB $)$ & $t_{\mathrm{SF}}(\mathrm{GCM})$ \\
\hline \hline$E_{\mathrm{Min}}$ & $0.81 \times 10^{43}$ & $0.70 \times 10^{30}$ \\
$S_{\mathrm{Min}}\left(Q_{20}, \Delta N^{2}\right)$ & $0.18 \times 10^{23}$ & $0.21 \times 10^{19}$ \\
$S_{\mathrm{Min}}\left(Q_{20}, Q_{30}\right)$ & $0.44 \times 10^{42}$ & $0.64 \times 10^{29}$ \\
$S_{\mathrm{Min}}\left(Q_{20}, Q_{40}\right)$ & $0.12 \times 10^{43}$ & $0.10 \times 10^{29}$ \\
\hline \hline
\end{tabular}

Table I. Spontaneous fission lifetimes computed with the ATDHFB and GCM approximations to the collective inertias. Results for different sets of collective variables used to search for the minimum of the action are given.

conclusion of that work is that minimum energy and minimum action results are essentially the same. Obviously, the result is not in contradiction with ours as different degrees of freedom are considered. In order to further investigate this aspect we have carried out action minimization calculations considering the pairs $\left(Q_{20}, Q_{30}\right)$ and $\left(Q_{20}\right.$, $\left.Q_{40}\right)$ of collective variables to explore the minimum action trajectory. The results obtained with BCPM are summarized in Table I. When compared with the results obtained using pairing correlations as a relevant collective variable in the action, the ones obtained considering $Q_{30}$ and $Q_{40}$ show a negligible impact being essentially the same as the ones obtained minimizing the energy. This can be understood as a consequence of the weak dependence of the collective inertia $B\left(Q_{20}\right)$ with $Q_{30}$ and $Q_{40}$. The conclusion is also evident, only the pairing degree of freedom is relevant for the action minimization calculation due to the dependence of the inertia with the inverse of the pairing gap.

Finally, we show in Table [I] a summary of the $t_{\mathrm{SF}}$ obtained minimizing the action and using the two EDF considered and the two variants of the collective inertia.
When compared with the results obtained minimizing the energy [25, 26, 31], the action minimization results show a much better agreement with experiment as well as a much reduced dispersion with the interaction used and the variant of the collective mass considered. This is a very important result as the theoretical interpretation of fission was hampered by the large variability on the $t_{\mathrm{SF}}$ values depending on the pairing strength.

\begin{tabular}{cccccc}
\hline \hline \multicolumn{2}{c}{ Gogny D1M } & \multicolumn{2}{c}{ BCPM } & Exp \\
$S_{\min }(\mathrm{A})$ & $S_{\min }(\mathrm{G})$ & $S_{\min }(\mathrm{A})$ & $S_{\min }(\mathrm{G})$ & \\
\hline \hline${ }^{232} \mathrm{U}$ & $5.4 \times 10^{20}$ & $1.5 \times 10^{17}$ & $8.6 \times 10^{19}$ & $8.2 \times 10^{16}$ & $2.5 \times 10^{21}$ \\
${ }^{234} \mathrm{U}$ & $7.3 \times 10^{21}$ & $2.7 \times 10^{18}$ & $1.7 \times 10^{22}$ & $2.1 \times 10^{18}$ & $4.7 \times 10^{23}$ \\
${ }^{236} \mathrm{U} 2.8 \times 10^{23}$ & $9.9 \times 10^{19}$ & $1.9 \times 10^{22}$ & $1.2 \times 10^{18}$ & $7.8 \times 10^{23}$ \\
${ }^{238} \mathrm{U} 1.6 \times 10^{24}$ & $6.7 \times 10^{20}$ & $6.1 \times 10^{21}$ & $6.2 \times 10^{17}$ & $2.6 \times 10^{23}$ \\
\hline \hline
\end{tabular}

Table II. Spontaneous fission lifetimes for several uranium isotopes obtained using the minimum action principle with the ATDHFB (A) and GCM $(G)$ variants of the collective inertias.

\section{CONCLUSIONS}

The main conclusion of the paper is that the scheme used to compute $t_{\mathrm{SF}}$ where the minimum action principle is used and the amount of pairing correlations is considered as collective variable gives results that strongly differ from the one where the energy is minimized to determine the fission path. The spontaneous fission lifetimes computed minimizing the action are several orders of magnitude smaller than the ones obtained from the traditional minimization of the energy. This decrease improves dramatically the agreement with the experimental data. In addition, the minimum action results show a weaker dependence with the ingredients of the calculation than the minimum energy ones. This is an important result for the credibility of mean field techniques to reproduce fission dynamics observables.

It is also shown that considering other variables in the minimization of the action like the multipole moments $Q_{30}$ and $Q_{40}$ has little impact on the results being equivalent to the energy minimization ones.

\section{ACKNOWLEDGMENTS}

Work supported in part by MICINN grants Nos. FPA2012-34694, FIS2012-34479 and by the ConsoliderIngenio 2010 program MULTIDARK CSD2009-00064. This work was initiated while one of the authors (LMR) participated at the INT13-3 program. The warm hospitality of the Institute for Nuclear Theory and the University of Washington is greatly acknowledged. 
[1] H.J. Krappe and K. Pomorski, Theory of Nuclear Fission, Lect. Notes in Phys. 838 (2012).

[2] D.N. Poenaru, Nuclear decay modes, Institute of Physics Publishing (1996).

[3] Hans J. Specht, Rev. Mod. Phys. 46, 773 (1974).

[4] S. Björnholm and J. E. Lynn, Rev. Mod. Phys. 52, 725 (1980).

[5] "Fifty Years of Fission", Nucl. Phys. A502 (1989).

[6] M. Brack, J. Damgaard, A.S. Jensen, H.C. Pauli, V.M. Strutinsky and C.Y. Wong, Rev. Mod. Phys. 44, 320 (1972).

[7] J.F. Berger, M. Girod and D. Gogny. Nucl. Phys. A428, 23c (1984).

[8] J. Bartel, P. Quentin, M. Brack, C. Guet and H.B. Häkansson, Nucl. Phys. A386, 79 (1982).

[9] N. Nikolov, N. Schunck, W. Nazarewicz, M. Bender and J. Pei, Phys. Rev. C83, 034305 (2011).

[10] J. McDonnell, N. Schunck and W. Nazarewicz, Fission and Properties of Neutron-Rich Nuclei (World Scientific, Singapore) p 597 (2013).

[11] E. Yuldashbaeva, J. Libert, P. Quentin, and M. Girod, Phys. Lett. B 461, 1 (1999).

[12] A. Baran, J.A. Sheikh, J. Dobaczewski, W. Nazarewicz, and A. Staszczak, Phys Rev C84, 054321 (2011).

[13] P. Ring and P. Shuck, The Nuclear Many Body Problem (Springer-Verlag Edt. Berlin, 1980).

[14] M. Bender, P.-H. Heenen, and P.-G. Reinhard, Rev. Mod. Phys. 75, 121 (2003).

[15] J. Erler, K. Langanke, H.P. Loens, G. Martinez-Pinedo, and P.-G. Reinhard Phys. Rev. C85, 025802 (2012).

[16] A. Staszczak, A. Baran and W. Nazarewicz, Phys. Rev. C 87, 024320 (2013).

[17] J.L. Egido and L.M. Robledo, Phys. Rev. Lett. 85, 1198 (2000).

[18] M. Warda, J.L. Egido, L.M. Robledo and K. Pomorski, Phys. Rev. C 66, 014310 (2002); Intl. J. of Mod. Phys. E 13, 169 (2004).

[19] J.-P. Delaroche, M. Girod, H. Goutte and J. Libert, Nucl. Phys. A 771, 103 (2006).

[20] N. Dubray, H. Goutte, and J.-P. Delaroche, Phys. Rev. C77, 014310 (2008).

[21] V. Martin and L.M. Robledo, Int. J. Mod. Phys. E 18, 788 (2009).

[22] S. Perez-Martin and L.M. Robledo, Int. J. Mod. Phys. E 18, 861 (2009).

[23] W. Younes and D. Gogny, Phys. Rev. C 80, 054313 (2009).

[24] M. Warda and L.M. Robledo, Phys. Rev. C 84, 044608
(2011).

[25] R. Rodríguez-Guzmán and L.M Robledo, Phys. Rev. C89, 054310 (2014).

[26] R. Rodríguez-Guzmán and L.M Robledo, arXiv: 1405.6784 .

[27] H. Abusara, A.V. Afanasjev and P. Ring, Phys. Rev. C82, 044303 (2010).

[28] B.N. Lu, E.G. Zhao and S.G. Zhou, Phys. Rev. C85, 011301 (2012).

[29] A.V. Afanasjev, Fission and Properties of Neutron-Rich Nuclei (World Scientific, Singapore) p 303 (2013).

[30] M. Baldo, L.M. Robledo, P. Schuck and X. Viñas, Physical Review C87, 064305 (2013).

[31] S.A. Giuliani and L.M Robledo, Phys. Rev. C 88, 054325 (2013).

[32] A. Baran, Phys. Lett. B76, 8 (1978).

[33] J. Sadhukhan, K. Mazurek, A. Baran, J. Dobaczewski, W. Nazarewicz and J.A. Sheikh Phys. Rev. C 88, 064314 (2013).

[34] L.G. Moretto, and R.P. Babinet, Phys. Lett. B49, 147 (1974).

[35] G.F. Bertsch and H. Flocard, Phys. Rev. C 43, 2200 (1991).

[36] D.R. Bès, R.A. Broglia, R.P.J. Perazzo, and K. Kumar, Nucl Phys A143, 1 (1970).

[37] A. Góźdź, K. Pomorski, M. Brack and E. Werner, Nucl Phys A442, 50 (1985).

[38] A. Staszczak, A. Baran, K. Pomorski and K. Böning Phys. Lett. B161, 227 (1978).

[39] K. Pomorski, Int. J. of Mod. Phys. E 16, 237 (2007).

[40] J. Decharge and D. Gogny, Phys. Rev. C 21, 1568 (1980).

[41] S. Goriely, S. Hilaire, M. Girod, and S. Perú, Phys. Rev. Lett. 102, 242501 (2009).

[42] J. Libert, M. Girod, and J.-P. Delaroche, Phys. Rev. C60, 054301 (1999).

[43] J.L Egido and L.M. Robledo, Lecture Notes in Physics 641, 269 (2004).

[44] L.M. Robledo, HFBaxial computer code (2002).

[45] L.M. Robledo and G.F. Bertsch, Phys. Rev C84, 014312 (2011).

[46] L.M. Robledo, R. R. Rodríguez-Guzmán and P. Sarriguren Phys Rev C 78, 034314 (2008).

[47] R. Rodríguez-Guzmán, P. Sarriguren, L.M. Robledo, S. Perez-Martin Phys. Lett. B 691, 202 (2010).

[48] R. Rodríguez-Guzmán, P. Sarriguren, L.M. Robledo, and J.E. García-Ramos Phys. Rev. C 81, 024310 (2010).

[49] L.M. Robledo and G.F. Bertsch, Phys. Rev. C84, 054302 (2011). 